\title{
Influence of Seed Treatments and Packaging Materials on Seed Quality Parameters during Storage in Buck Wheat (Fagopyrum esculentum L.)
}

\author{
Chelakani Srujana*, Ajay Kumar Pandey and A. K. Chaurasia
}

Naini Agricultural Institute, SHUATS, Prayagraj-211007, Uttar Pradesh, India

*Corresponding author

\section{A B S T R A C T}

\begin{tabular}{|l|}
\hline K e y w o r d s \\
Buckwheat, Neem \\
oil, Carbendazim, \\
Polythene bag, \\
Cloth bag
\end{tabular}

\begin{abstract}
A storage experiment was conducted to understand the effect of seed treatments and packaging materials on seed quality parameters of Buckwheat genotypes during storage at Seed Testing Laboratory, Department of Genetics and plant Breeding, SHUATS, Prayagraj, U.P during 2019-2020.The Experiment. Consists of five genotypes viz, IC13140(G1), IC-13143(G2), IC-13144(G3), IC-13191(G4) -IC-13412(G5). Seeds were treated with Neem oil at $5 \mathrm{ml} / \mathrm{kg}\left(\mathrm{T}_{1}\right)$ and carbendazim at $2 \mathrm{~g} / \mathrm{kg}\left(\mathrm{T}_{2}\right)$ and seed alone with control ( untreated $\mathrm{T}_{0}$ ) and packed in polythene bag $\left(\mathrm{C}_{1}\right)$ and cloth bag $\left(\mathrm{C}_{2}\right)$ and maintained for 9 months under ambient conditions. The results clearly revealed that seeds of IC-13143 (G2) treated with neem oil which were stored in polythene bag were found high germination percent $(82.75 \%)$, root length $(12.25 \mathrm{~cm})$, shoot length $(9.97 \mathrm{~cm})$, seedling length $(22.22 \mathrm{~cm})$, fresh weight $(0.8 \mathrm{~g})$, dry weight $(0.07 \mathrm{~g})$, vigour index-I (1839.2), vigour index-II (5.38) and viability (70).
\end{abstract}

\section{Introduction}

Buckwheat (Fagropyrum esculentum $\mathrm{L}$ ) is an annual crop, it is a pseudo cereal but its grains belong to cereals because of their similar use and chemical composition (Campbell, 1997). It is a multipurpose crop. The tender shoots are used as leafy vegetables. Buckwheat grains are an important source of microelements, such as: $\mathrm{Zn}, \mathrm{Cu}, \mathrm{Mn}, \mathrm{Se}$ (Stibilj et al., 2004), and macro elements: K, $\mathrm{Na}, \mathrm{Ca}, \mathrm{Mg}$ (Wei et al., 2003).

Losses in seed quality occur during field weathering, harvesting and storage due to which seeds get damaged. If seeds are exposed to high temperature and high humidity, the incidence of microflora is mainly responsible for the degradation of protein and other food reserve resulting in reduction in viability, vigour and germination. To combat these factors, it is better to store the seeds in moisture impervious containers like polythene bag and some are moisture pervious container like cloth bag to maintain the quality for longer period. Indiscriminate use of Chemical and their residual toxicity adversely affect the seed quality. Many of synthetic chemicals look effective but they are not readily degradable physically or biologically which yield more toxic residues. Hence, the feasible approach is the treatment 
of seeds with botanicals which are safe, economical, eco-friendly, cheap, easily locally available and non-harmful to seeds, animals and human beings. It will be of immense use to the farming community. Therefore, the present investigation was taken up to study the effect of seed treatments on seed quality characters of buckwheat genotypes during storage.

\section{Materials and Methods}

The experiment was laid down in CRD with four replications. The Buckwheat seeds IC$13140\left(\mathrm{G}_{1}\right)$, IC-13143 $\left(\mathrm{G}_{2}\right)$, IC-13144 $\left(\mathrm{G}_{3}\right)$, IC-13191 $\left(\mathrm{G}_{4}\right)$ and IC-13412 $\left(\mathrm{G}_{5}\right)$ were dried to bring down the moisture content to 10-12 $\%$ before proceeding for storage. Seed was treated with Neem oil at $5 \mathrm{ml} / \mathrm{kg}\left(\mathrm{T}_{1}\right)$, Carbendazim at $2 \mathrm{~g} / \mathrm{kg} \mathrm{T}$ ) with local control $\left(\mathrm{T}_{0}\right)$ stored for 9 months at ambient room temperature in the seed testing Laboratory of department of Genetics and Plant Breeding, Naini Agricultural Institute, SHUATS, Allahabad with packed in packaging materials viz polythene bag, cloth bag.

\section{Results and Discussion}

\section{Germination percent}

The significant differences with respect to treatments were observed in Table 1. The seeds of IC-13143 $\left(\mathrm{G}_{2}\right)$ genotype treated with biocide (Neem oil at $5 \mathrm{ml} / \mathrm{kg}$ ) stored in polythene bag recorded maximum germination percent $(82.75 \%)$ followed by seeds of IC-13140 $\left(\mathrm{G}_{1}\right)(82.70 \%)$ treated with biocide (Neem oil at $5 \mathrm{ml} / \mathrm{kg}$ ). Now a day's botanicals are widely used in place of fungicides and pesticides for seed treatment to protect seeds against pests and diseases during storage. The present results are in close agreement with the reports of Hanegave (2009) in wheat.

\section{Root length}

The effect of seed treatments on root length (cm) was significant after 9 months of storage periods (Table 1). Results revealed that the seeds of IC-13143 $\left(\mathrm{G}_{2}\right)$ among all the genotypes and treated with biocide (Neem oil at $5 \mathrm{ml} / \mathrm{kg}$ ) stored in polythene bag recorded maximum mean root length $(12.25 \mathrm{~cm})$ followed by seeds of IC-13140 $\left(\mathrm{G}_{1}\right)(12.05$ $\mathrm{cm}$ ) treated with biocide (Neem oil at 5 $\mathrm{ml} / \mathrm{kg}$ ).

The beneficial influence noticed with these botanicals may be related to their protective nature, antifungal, insecticidal property besides synergistic effect with amino acids in germinating seeds to protect the root apical meristems tissue from damage and enhance longitudinal root growth, Dileepkumar et al., (2009) in cowpea; Raikar et al., (2011) in Rice; Nargis and Thiagarajan (1991); Paul and Mishra (1994); Samuel et al., (2008).

\section{Shoot length}

The effect of seed treatments on shoot length (cm) was significant after 9 months of storage periods (Table 1). Results revealed that the seeds of IC-13143 $\left(\mathrm{G}_{2}\right)$ among all the genotypes and treated with biocide (Neem oil at $5 \mathrm{ml} / \mathrm{kg}$ ) stored in polythene bag recorded maximum mean shoot length $(9.97 \mathrm{~cm})$ followed by seeds of IC-13140 $\left(\mathrm{G}_{1}\right)(9.95 \mathrm{~cm})$ treated with biocide (Neem oil at $5 \mathrm{ml} / \mathrm{kg}$ ).

Similar beneficial results with other different botanicals on germination were reported by Ntonifor et al., (2011) in rice.

\section{Seedling length}

The effect of seed treatments on seedling length $(\mathrm{cm})$ was significant after 9 months of storage periods (Table 2). 
Table.1 Effect of different treatments on Germination \%, Root length and Shoot length of various genotypes of Buckwheat (Fagopyrum esculentum L.) stored in Polythene bags and Cloth bags

\begin{tabular}{|c|c|c|c|c|c|c|c|c|c|c|c|c|c|c|c|c|c|c|}
\hline \multirow{3}{*}{ Variety } & \multicolumn{6}{|c|}{ Germination \% } & \multicolumn{6}{|c|}{ Root Length } & \multicolumn{6}{|c|}{ Shoot Length } \\
\hline & \multicolumn{2}{|c|}{3 Month } & \multicolumn{2}{|c|}{6 Month } & \multicolumn{2}{|c|}{9 Month } & \multicolumn{2}{|c|}{3 Month } & \multicolumn{2}{|c|}{6 Month } & \multicolumn{2}{|c|}{9 Month } & \multicolumn{2}{|c|}{3 Month } & \multicolumn{2}{|c|}{6 Month } & \multicolumn{2}{|c|}{9 Month } \\
\hline & $\mathrm{C}_{1}$ & $\mathrm{C}_{2}$ & $\mathrm{C}_{1}$ & $\mathrm{C}_{2}$ & $\mathrm{C}_{1}$ & $\mathrm{C}_{2}$ & $\mathrm{C}_{1}$ & $\mathrm{C}_{2}$ & $\mathrm{C}_{1}$ & $\mathrm{C}_{2}$ & $\mathrm{C}_{1}$ & $\mathrm{C}_{2}$ & $\mathrm{C}_{1}$ & $\mathrm{C}_{2}$ & $\mathrm{C}_{1}$ & $\mathrm{C}_{2}$ & $\mathrm{C}_{1}$ & $\mathrm{C}_{2}$ \\
\hline $\mathbf{T}_{0} \mathbf{G}_{1}$ & 91.25 & 91.25 & 80.5 & 80.5 & 77.25 & 77.25 & 13.67 & 12.57 & 10.75 & 9.85 & 8.85 & 7.77 & 9.4 & 8.3 & 8.05 & 7 & 7.175 & 6.075 \\
\hline $\mathbf{T}_{0} \mathbf{G}_{2}$ & 96.75 & 96.5 & 77.25 & 77.25 & 78.25 & 78.25 & 10.17 & 9.07 & 8.15 & 7.1 & 5.75 & 5.5 & 8.85 & 7.75 & 7.95 & 6.9 & 6.95 & 5.9 \\
\hline $\mathbf{T}_{0} \mathbf{G}_{3}$ & 96 & 95 & 78.25 & 78.25 & 74 & 74 & 7.5 & 7.3 & 7.05 & 6 & 6.25 & 5.2 & 9.725 & 8.625 & 8.95 & 7.9 & 6.975 & 5.95 \\
\hline $\mathbf{T}_{0} \mathbf{G}_{4}$ & 93.5 & 93.25 & 75.25 & 75.25 & 72.5 & 72.5 & 12.95 & 11.9 & 9.25 & 8.15 & 7.55 & 6.5 & 9.5 & 8.4 & 8.4 & 7.3 & 6.9 & 5.8 \\
\hline $\mathbf{T}_{0} \mathbf{G}_{5}$ & 98.75 & 99 & 92.5 & 92.5 & 73.25 & 73 & 11.25 & 10.25 & 9.15 & 8.1 & 7.97 & 6.95 & 8.45 & 7.35 & 7.95 & 6.9 & 6.175 & 5.075 \\
\hline $\mathbf{T}_{1} \mathbf{G}_{1}$ & 97.75 & 97.75 & 88.5 & 87 & 82.70 & 77.25 & 13.94 & 12.9 & 12.55 & 11.45 & 12.05 & 11 & 11.6 & 10.5 & 10.1 & 9.025 & 9.95 & 8.7 \\
\hline $\mathbf{T}_{1} \mathbf{G}_{2}$ & 98.5 & 97.5 & 84.5 & 83 & 82.75 & 78.25 & 14.75 & 13.7 & 12.85 & 11.75 & 12.25 & 11.12 & 11.5 & 10.4 & 10.1 & 9 & 9.975 & 8.9 \\
\hline $\mathbf{T}_{1} \mathbf{G}_{3}$ & 98.25 & 97.25 & 92.5 & 91 & 80.25 & 74 & 14.82 & 13.8 & 12.17 & 11.07 & 11.97 & 10.95 & 11.95 & 10.9 & 10.12 & 9.025 & 8.95 & 7.9 \\
\hline $\mathbf{T}_{1} \mathbf{G}_{4}$ & 96.5 & 95.75 & 91.5 & 90 & 80.5 & 72.5 & 13.45 & 12.4 & 11.85 & 10.8 & 11.55 & 10.5 & 11.6 & 10.5 & 9.95 & 8.9 & 8.8 & 7.7 \\
\hline $\mathbf{T}_{1} \mathbf{G}_{5}$ & 98 & 97 & 93.25 & 92.25 & 80 & 73.25 & 13.8 & 12.8 & 11.65 & 10.6 & 11.17 & 10.1 & 10.95 & 9.9 & 10.1 & 9 & 8.95 & 7.9 \\
\hline $\mathbf{T}_{2} \mathbf{G}_{1}$ & 97.75 & 96.75 & 91.5 & 90.5 & 78.25 & 77.5 & 13.65 & 12.6 & 11.95 & 10.85 & 9.85 & 8.8 & 10.3 & 9.2 & 9.1 & 8 & 7.95 & 6.9 \\
\hline $\mathbf{T}_{2} \mathbf{G}_{2}$ & 97 & 96 & 84.5 & 83.75 & 79 & 77.5 & 13.05 & 12 & 9.15 & 8.05 & 6.85 & 5.75 & 9.95 & 8.9 & 8.95 & 7.9 & 7.6 & 6.5 \\
\hline $\mathbf{T}_{2} \mathbf{G}_{3}$ & 93.25 & 92.5 & 91.5 & 90.5 & 74.25 & 73.25 & 11.67 & 10.57 & 8.25 & 7.1 & 7.32 & 6.25 & 10.9 & 9.8 & 9.4 & 8.3 & 7.975 & 6.95 \\
\hline $\mathbf{T}_{2} \mathbf{G}_{4}$ & 98.5 & 97.5 & 75.5 & 74 & 73.5 & 72 & 13.12 & 12.02 & 10.55 & 9.45 & 8.75 & 7.7 & 10.85 & 8.75 & 9.6 & 8.5 & 7.9 & 6.8 \\
\hline $\mathbf{T}_{2} \mathbf{G}_{5}$ & 97.5 & 96.5 & 87.5 & 86.5 & 75 & 73.75 & 12.45 & 11.4 & 10.97 & 9.95 & 10.5 & 9.5 & 9.9 & 8.8 & 9.15 & 8.05 & 7.95 & 6.9 \\
\hline Mean & 96.61 & 95.96 & 85.63 & 84.81 & 77.43 & 74.95 & 12.68 & 11.68 & 10.41 & 9.35 & 9.24 & 8.24 & 10.36 & 9.20 & 9.19 & 8.11 & 8.01 & 6.93 \\
\hline SE \pm & 0.64 & 0.42 & 1.22 & 0.53 & 1.01 & 0.6 & 0.49 & 0.20 & 0.38 & 0.17 & 0.45 & 0.15 & 0.072 & 0.06 & 0.062 & 0.03 & 0.03 & 0.08 \\
\hline $\mathrm{CV}$ & 1.32 & 0.33 & 2.84 & 1.25 & 2.60 & 1.60 & 7.88 & 3.45 & 7.38 & 3.72 & 9.94 & 3.74 & 1.40 & 1.51 & 1.35 & 0.77 & 0.77 & 2.50 \\
\hline CD & 0.91 & 0.60 & 1.73 & 0.75 & 1.44 & 0.85 & 0.71 & 0.28 & 0.54 & 0.24 & 0.65 & 0.21 & 0.104 & 0.09 & 0.088 & 0.04 & 0.04 & 0.12 \\
\hline
\end{tabular}


Table.2 Effect of different treatments on Seedling length, Fresh weight and Dry weight of various genotypes of Buckwheat (Fagopyrum esculentum L.) stored in Polythene bags and Cloth bags

\begin{tabular}{|c|c|c|c|c|c|c|c|c|c|c|c|c|c|c|c|c|c|c|}
\hline \multirow{3}{*}{$\begin{array}{l}\text { Variet } \\
\mathbf{y}\end{array}$} & \multicolumn{6}{|c|}{ Seedling Length } & \multicolumn{6}{|c|}{ Fresh Weight } & \multicolumn{6}{|c|}{ Dry Weight } \\
\hline & \multicolumn{2}{|c|}{3 Month } & \multicolumn{2}{|c|}{6 Month } & \multicolumn{2}{|c|}{9 Month } & \multicolumn{2}{|c|}{3 Month } & \multicolumn{2}{|c|}{6 Month } & \multicolumn{2}{|c|}{9 Month } & \multicolumn{2}{|c|}{3 Month } & \multicolumn{2}{|c|}{6 Month } & \multicolumn{2}{|c|}{9 Month } \\
\hline & $\mathrm{C}_{1}$ & $\mathrm{C}_{2}$ & $\mathrm{C}_{1}$ & $\mathrm{C}_{2}$ & $\mathrm{C}_{1}$ & $\mathrm{C}_{2}$ & $\mathrm{C}_{1}$ & $\mathrm{C}_{2}$ & $\mathrm{C}_{1}$ & $\mathrm{C}_{2}$ & $\mathrm{C}_{1}$ & $\mathrm{C}_{2}$ & $\mathrm{C}_{1}$ & $\mathrm{C}_{2}$ & $\mathrm{C}_{1}$ & $\mathrm{C}_{2}$ & $\mathrm{C}_{1}$ & $\mathrm{C}_{2}$ \\
\hline $\mathbf{T}_{0} \mathbf{G}_{1}$ & 23.07 & 20.87 & 18.8 & 16.85 & 16.02 & 13.85 & 0.95 & 0.8 & 0.57 & 0.475 & 0.5 & 0.4 & 0.052 & 0.04 & 0.04 & 0.022 & 0.02 & 0.012 \\
\hline $\mathbf{T}_{0} \mathbf{G}_{2}$ & 19.02 & 16.82 & 16.1 & 14 & 12.7 & 11.4 & 0.85 & 0.8 & 0.5 & 0.4 & 0.47 & 0.37 & 0.052 & 0.032 & 0.03 & 0.02 & 0.02 & 0.015 \\
\hline $\mathbf{T}_{0} \mathbf{G}_{3}$ & 17.22 & 15.92 & 16 & 13.9 & 13.22 & 11.15 & 0.55 & 0.4 & 0.35 & 0.25 & 0.3 & 0.2 & 0.055 & 0.045 & 0.04 & 0.03 & 0.02 & 0.012 \\
\hline $\mathbf{T}_{0} \mathbf{G}_{4}$ & 22.45 & 20.3 & 17.65 & 15.45 & 14.45 & 12.3 & 0.95 & 0.8 & 0.6 & 0.5 & 0.42 & 0.32 & 0.052 & 0.037 & 0.04 & 0.025 & 0.02 & 0.012 \\
\hline $\mathbf{T}_{\mathbf{0}} \mathbf{G}_{5}$ & 19.7 & 17.6 & 17.1 & 15 & 14.15 & 12.02 & 0.6 & 0.47 & 0.4 & 0.3 & 0.35 & 0.25 & 0.065 & 0.055 & 0.04 & 0.027 & 0.03 & 0.015 \\
\hline $\mathbf{T}_{1} \mathbf{G}_{1}$ & 25.54 & 23.4 & 22.65 & 20.47 & 22 & 19.7 & 1.15 & 1 & 0.95 & 0.85 & 0.7 & 0.6 & 0.085 & 0.082 & 0.07 & 0.057 & 0.06 & 0.045 \\
\hline $\mathbf{T}_{1} \mathbf{G}_{2}$ & 26.25 & 24.1 & 22.95 & 20.75 & 22.22 & 20.02 & 1.15 & 1.05 & 0.98 & 0.87 & 0.8 & 0.7 & 0.085 & 0.08 & 0.07 & 0.057 & 0.07 & 0.05 \\
\hline $\mathbf{T}_{1} \mathbf{G}_{3}$ & 26.77 & 24.7 & 22.3 & 20.1 & 20.92 & 18.85 & 1.05 & 1 & 0.9 & 0.8 & 0.67 & 0.57 & 0.085 & 0.077 & 0.07 & 0.057 & 0.06 & 0.043 \\
\hline $\mathbf{T}_{1} \mathbf{G}_{4}$ & 25.05 & 22.9 & 21.8 & 19.7 & 20.35 & 18.2 & 0.99 & 0.87 & 0.8 & 0.7 & 0.65 & 0.55 & 0.09 & 0.082 & 0.08 & 0.07 & 0.05 & 0.035 \\
\hline $\mathbf{T}_{1} \mathbf{G}_{5}$ & 24.75 & 22.7 & 21.75 & 19.6 & 20.12 & 18 & 0.98 & 0.85 & 0.75 & 0.65 & 0.65 & 0.57 & 0.085 & 0.075 & 0.07 & 0.05 & 0.05 & 0.035 \\
\hline $\mathbf{T}_{2} \mathbf{G}_{1}$ & 23.95 & 21.8 & 21.05 & 18.85 & 17.8 & 15.7 & 0.99 & 0.87 & 0.65 & 0.55 & 0.55 & 0.45 & 0.054 & 0.042 & 0.04 & 0.032 & 0.03 & 0.017 \\
\hline $\mathbf{T}_{\mathbf{2}} \mathbf{G}_{2}$ & 23 & 20.9 & 18.1 & 15.95 & 14.45 & 12.25 & 0.87 & 0.75 & 0.55 & 0.45 & 0.5 & 0.4 & 0.048 & 0.04 & 0.03 & 0.02 & 0.02 & 0.021 \\
\hline $\mathbf{T}_{\mathbf{2}} \mathbf{G}_{\mathbf{3}}$ & 22.57 & 20.37 & 17.65 & 15.4 & 15.3 & 13.2 & 0.57 & 0.45 & 0.4 & 0.3 & 0.35 & 0.25 & 0.057 & 0.05 & 0.04 & 0.03 & 0.03 & 0.022 \\
\hline $\mathbf{T}_{2} \mathbf{G}_{4}$ & 23.97 & 20.77 & 20.15 & 17.95 & 16.65 & 14.5 & 0.99 & 0.87 & 0.7 & 0.6 & 0.5 & 0.4 & 0.05 & 0.04 & 0.04 & 0.025 & 0.02 & 0.022 \\
\hline $\mathbf{T}_{\mathbf{2}} \mathbf{G}_{5}$ & 22.35 & 20.2 & 20.12 & 18 & 18.45 & 16.4 & 0.65 & 0.5 & 0.45 & 0.35 & 0.4 & 0.3 & 0.067 & 0.06 & 0.04 & 0.027 & 0.03 & 0.015 \\
\hline Mean & 23.04 & 20.89 & 19.61 & 17.46 & 17.25 & 15.17 & 0.88 & 0.76 & 0.63 & 0.53 & 0.52 & 0.42 & 0.065 & 0.056 & 0.05 & 0.036 & 0.03 & 0.024 \\
\hline $\mathrm{SE} \pm$ & 0.51 & 0.20 & 0.39 & 0.26 & 0.45 & 0.17 & 0.05 & 0.06 & 0.05 & 0.04 & 0.05 & 0.05 & 0.0035 & 0.003 & 0.004 & 0.004 & 0.005 & 0.005 \\
\hline $\mathrm{CV}$ & 4.43 & 1.98 & 4.04 & 3.02 & 5.24 & 2.36 & 12.7 & 15.89 & 16.08 & 16.93 & 19.88 & 24.69 & 10.55 & 14.02 & 17.21 & 24.50 & 33.11 & 44.64 \\
\hline CD & 0.72 & 0.29 & 0.56 & 0.37 & 0.64 & 0.25 & 0.08 & 0.08 & 0.073 & 0.06 & 0.07 & 0.07 & 0.005 & 0.005 & 0.006 & 0.006 & 0.007 & 0.007 \\
\hline
\end{tabular}


Table.3 Effect of different treatments on Vigour Index-I, Vigour Index-II and Viability of various genotypes of Buckwheat (Fagopyrum esculentum L.) stored in Polythene bags and Cloth bags

\begin{tabular}{|c|c|c|c|c|c|c|c|c|c|c|c|c|c|c|c|c|c|c|}
\hline \multirow{3}{*}{$\begin{array}{l}\text { Varie } \\
\text { ty }\end{array}$} & \multicolumn{6}{|c|}{ Vigour Index-I } & \multicolumn{6}{|c|}{ Vigour Index-II } & \multicolumn{6}{|c|}{ Viability } \\
\hline & \multicolumn{2}{|c|}{3 Month } & \multicolumn{2}{|c|}{6 Month } & \multicolumn{2}{|c|}{9 Month } & \multicolumn{2}{|c|}{3 Month } & \multicolumn{2}{|c|}{6 Month } & \multicolumn{2}{|c|}{9 Month } & \multicolumn{2}{|c|}{3 Month } & \multicolumn{2}{|c|}{6 Month } & \multicolumn{2}{|c|}{9 Month } \\
\hline & $\mathrm{C}_{1}$ & $\mathrm{C}_{2}$ & $\mathrm{C}_{1}$ & $\mathrm{C}_{2}$ & $\mathrm{C}_{1}$ & $\mathrm{C}_{2}$ & $\mathrm{C}_{1}$ & $\mathrm{C}_{2}$ & $\mathrm{C}_{1}$ & $\mathrm{C}_{2}$ & $\mathrm{C}_{1}$ & $\mathrm{C}_{2}$ & $\mathrm{C}_{1}$ & $\mathrm{C}_{2}$ & $\mathrm{C}_{1}$ & $\mathrm{C}_{2}$ & $\mathrm{C}_{1}$ & $\mathrm{C}_{2}$ \\
\hline $\mathbf{T}_{0} \mathbf{G}_{1}$ & 2103.85 & 1904.7 & 1512.4 & 1356.42 & 1238.62 & 1069.7 & 4.79 & 3.64 & 3.435 & 1.81 & 1.82 & 0.96 & 82 & 70 & 62 & 50 & 46 & 34 \\
\hline $\mathbf{T}_{0} \mathbf{G}_{2}$ & 1840.5 & 1623.57 & 1244.5 & 1081.85 & 993.65 & 892.35 & 5.07 & 3.12 & 2.32 & 1.54 & 1.74 & 1.17 & 84 & 72 & 64 & 52 & 48 & 36 \\
\hline $\mathbf{T}_{0} \mathbf{G}_{3}$ & 1653.75 & 1512.87 & 1252.0 & 1087.67 & 978.2 & 825.02 & 5.27 & 4.27 & 3.32 & 2.34 & 1.29 & 0.92 & 86 & 74 & 62 & 50 & 50 & 38 \\
\hline $\mathbf{T}_{0} \mathbf{G}_{4}$ & 2099.1 & 1893.1 & 1327.1 & 1162.65 & 1048.65 & 891.75 & 4.91 & 3.49 & 3.192 & 1.88 & 1.63 & 0.90 & 88 & 76 & 62 & 50 & 52 & 40 \\
\hline $\mathbf{T}_{0} \mathbf{G}_{5}$ & 1944.9 & 1742.32 & 1580.6 & 1387.52 & 1036.15 & 877.95 & 6.41 & 5.44 & 3.69 & 2.545 & 1.84 & 1.09 & 82 & 70 & 64 & 52 & 45 & 32 \\
\hline $\mathbf{T}_{1} \mathbf{G}_{1}$ & 2497.73 & 2287.32 & 2005.7 & 1781.35 & 1820 & 1537.97 & 8.3 & 8.06 & 5.96 & 5.002 & 4.55 & 3.47 & 92 & 80 & 74 & 62 & 70 & 57 \\
\hline $\mathbf{T}_{1} \mathbf{G}_{2}$ & 2585.25 & 2349.97 & 1939.6 & 1722.05 & 1839.2 & 1551.37 & 8.36 & 7.79 & 6.12 & 4.772 & 5.38 & 3.91 & 88 & 76 & 76 & 64 & 70 & 58 \\
\hline $\mathbf{T}_{1} \mathbf{G}_{3}$ & 2631.3 & 2402.07 & 2063.2 & 1828.75 & 1678.52 & 1394.95 & 8.35 & 7.53 & 6.70 & 5.225 & 4.40 & 3.14 & 92 & 80 & 72 & 60 & 68 & 56 \\
\hline $\mathbf{T}_{1} \mathbf{G}_{4}$ & 2417.67 & 2192.57 & 1994.8 & 1773 & 1637.52 & 1319.8 & 8.68 & 7.9 & 7.09 & 6.305 & 3.99 & 2.53 & 94 & 82 & 76 & 65 & 68 & 56 \\
\hline $\mathbf{T}_{1} \mathbf{G}_{5}$ & 2424.42 & 2201.92 & 2028.1 & 1808.72 & 1610.12 & 1318.52 & 8.33 & 7.27 & 6.04 & 4.62 & 3.62 & 2.56 & 92 & 80 & 72 & 60 & 64 & 52 \\
\hline $\mathbf{T}_{2} \mathbf{G}_{1}$ & 2341.2 & 2109.15 & 1926.3 & 1705.9 & 1393.35 & 1216.82 & 5.35 & 4.11 & 3.88 & 2.942 & 1.94 & 1.36 & 88 & 76 & 68 & 56 & 52 & 40 \\
\hline $\mathbf{T}_{2} \mathbf{G}_{2}$ & 2231.025 & 2006.67 & 1529.9 & 1335.67 & 1141 & 949.37 & 4.70 & 3.83 & 2.75 & 1.672 & 1.58 & 1.66 & 90 & 78 & 70 & 58 & 56 & 44 \\
\hline $\mathbf{T}_{2} \mathbf{G}_{3}$ & 2104.7 & 1884.77 & 1615.0 & 1393.37 & 1135.57 & 966.75 & 5.36 & 4.62 & 3.89 & 2.712 & 1.23 & 1.65 & 88 & 76 & 68 & 56 & 58 & 46 \\
\hline $\mathbf{T}_{2} \mathbf{G}_{4}$ & 2361.47 & 2025.65 & 1521.9 & 1328.32 & 1222.57 & 1044.02 & 4.93 & 3.89 & 3.20 & 1.867 & 1.66 & 1.61 & 90 & 78 & 72 & 60 & 60 & 48 \\
\hline $\mathbf{T}_{2} \mathbf{G}_{5}$ & 2179.15 & 1949.15 & 1759.8 & 1556.75 & 1383.52 & 1209.27 & 6.58 & 5.78 & 3.71 & 2.3 & 1.87 & 1.10 & 92 & 78 & 70 & 58 & 54 & 42 \\
\hline Mean & 2227.7 & 2005.72 & 1686.75 & 1487.33 & 1343.77 & 1137.71 & 6.36 & 5.38 & 3.35 & 3.17 & 2.64 & 1.87 & 88.53 & 76.4 & 68.8 & 56.8 & 57.4 & 45.2 \\
\hline $\mathrm{SE} \pm$ & 49.42 & 22.64 & 40.88 & 23.29 & 34.73 & 18.70 & 0.32 & 0.37 & 0.37 & 0.39 & 0.43 & 0.47 & 0.52 & 0.46 & 0.46 & 0.35 & 0.39 & 0.41 \\
\hline $\mathrm{CV}$ & 4.43 & 2.25 & 4.84 & 3.13 & 5.16 & 3.28 & 10.35 & 13.84 & 16.98 & 24.69 & 33.16 & 44.74 & 1.19 & 1.22 & 1.34 & 1.24 & 1.37 & 1.84 \\
\hline CD & 70.52 & 32.3 & 58.34 & 33.23 & 49.56 & 26.69 & 0.47 & 0.53 & 0.52 & 0.55 & 0.62 & 0.59 & 0.75 & 0.66 & 0.66 & 0.50 & 0.56 & 0.59 \\
\hline
\end{tabular}


Results revealed that the seeds of IC-13143 $\left(\mathrm{G}_{2}\right)$ among all the genotypes and treated with biocide (Neem oil at $5 \mathrm{ml} / \mathrm{kg}$ ) stored in polythene bag recorded maximum mean seedling length $(22.22 \mathrm{~cm})$ followed by seeds of IC-13140 $\left(\mathrm{G}_{1}\right)(22.0 \mathrm{~cm})$ treated with biocide (Neem oil at $5 \mathrm{ml} / \mathrm{kg}$ ).

\section{Fresh Weight}

The effect of seed treatments on Fresh weight (g) was significant after 9 months of storage periods (Table 2). Results revealed that the seeds of IC-13143 $\left(\mathrm{G}_{2}\right)$ among all the genotypes and treated with biocide (Neem oil at $5 \mathrm{ml} / \mathrm{kg}$ ) stored in polythene bag recorded maximum mean fresh weight $(0.8 \mathrm{~g})$ followed by seeds of IC-13140 $\left(\mathrm{G}_{1}\right)(0.7 \mathrm{~g})$ treated with biocide (Neem oil at $5 \mathrm{ml} / \mathrm{kg}$ ).

\section{Dry weight}

The effect of seed treatments on dry weight (cm) was significant after 9 months of storage periods (Table 2). Results revealed that the seeds of IC-13143 $\left(\mathrm{G}_{2}\right)$ among all the genotypes and treated with biocide (Neem oil at $5 \mathrm{ml} / \mathrm{kg}$ ) stored in polythene bag recorded maximum mean dry weight $(0.07 \mathrm{~g})$ followed by seeds of IC-13140 $\left(\mathrm{G}_{1}\right)(0.06 \mathrm{~g})$ and $\mathrm{IC}$ $13144\left(\mathrm{G}_{3}\right)(0.06 \mathrm{~g})$ treated with biocide (Neem oil at $5 \mathrm{ml} / \mathrm{kg}$ ).

\section{Vigour Index- I}

The effect of seed treatments on Vigour Index- I was significant after 9 months of storage periods (Table 3). Results revealed that the seeds of IC-13143 $\left(\mathrm{G}_{2}\right)$ among all the genotypes and treated with biocide (Neem oil at $5 \mathrm{ml} / \mathrm{kg}$ ) stored in polythene bag recorded maximum mean Vigour Index- I (1839.2) followed by seeds of IC-13140 $\left(\mathrm{G}_{1}\right)(1820)$ treated with biocide (Neem oil at $5 \mathrm{ml} / \mathrm{kg}$ ). The results are similar to the findings of seedling of Asawalam et al., (2008). Seed quality is judged by seedling vigour parameters like root and shoot length, vigour index and seedling dry weight. Generally, higher the seedling length, vigour index and seedling dry weight, higher is the seed quality.

\section{Vigour Index- II}

The effect of seed treatments on Vigour Index- II was significant after 9 months of storage periods (Table 3). Results revealed that the seeds of IC-13143 $\left(\mathrm{G}_{2}\right)$ among all the genotypes and treated with biocide (Neem oil at $5 \mathrm{ml} / \mathrm{kg}$ ) stored in polythene bag recorded maximum mean Vigour Index- II (5.38) followed by seeds of IC-13140 $\left(\mathrm{G}_{1}\right)$ (4.55) treated with biocide (Neem oil at $5 \mathrm{ml} / \mathrm{kg}$ ).

\section{Viability}

The effect of seed treatments on Viability was significant after 9 months of storage periods (Table 3). Results revealed that the seeds of IC-13143 $\left(\mathrm{G}_{2}\right)$ and $\mathrm{IC}-13140\left(\mathrm{G}_{1}\right)$ among all the genotypes and treated with biocide (Neem oil at $5 \mathrm{ml} / \mathrm{kg}$ ) stored in polythene bag recorded maximum mean Viability (70.00) followed by seeds of IC-13144 $\left(\mathrm{G}_{3}\right)(68)$ and treated with biocide (Neem oil at $5 \mathrm{ml} / \mathrm{kg}$ ).

It is concluded that from the present investigation in buckwheat of all treated seeds, seeds of IC-13143 $\left(\mathrm{G}_{2}\right)$ treated with neem oil stored in polythene bag recorded significantly higher seed quality parameters. Seed treatments have a major role in protecting the seed during storage and can also play an important role in achieving uniform seedling emergence under certain conditions.

\section{Acknowledgement}

We sincerely thankful to Department of Genetics and Plant Breeding, Naini Agricultural Institute, SHUATS, Prayagraj for 
providing laboratory facilities and assistance in conducting this research.

\section{References}

Agrawal, P. K., 1974, Storage studies on maize seeds. Bul. of Grain Technol., 12: 109-112.

Agrawal, P. K., 1980, Relative storability of seeds of ten species under ambient conditions. Seed Res., 8: 94-99.

Arati, P., 2000, Influence of containers and seed treatments on storability of chickpea. M.Sc. (Agri.) Thesis, Univ. of Agril. Sci., Dharwad, Karnataka, India.

Asawalam, E. F., Emosairue, S. O. and Hassanali, A., 2008, Contribution of different constituents to the toxicity of the essential oil constituents of Vernonia amydalina (Compositae) and Xylopia aetiopica (Annonaceae) on maize weevil, Sitophilus zeamais Motschulsky (Coleoptera: Curculionidae). Nig. J. Entomol, 23: 30-33.

Campbell C.G. (1997) Buckwheat fagopyrum esculentum Moench, International Plant Genetic Resources Institute, CAB International.

Dileepkumar, A. Masuthi, Vyakaranahal, B. S., Deshpande, V. K., 2009 Influence of pelleting with micronutrients and botanical on growth, seed yield and quality of vegetable cowpea. Kar. J. Agric. Sci., 22(4): 898-900.

Hanegave, A. S., 2009, Influence of seed invigouration and polymer coating on field performance and storability of maize (Zea mays L.) M. Sc. Thesis, Univ. Agri. Sci., Dharwad, India.

Nargis, S., and Thiagarajan, C. P., 1991. Storage studies with pelleted seeds of tomato Cv. PKM1. South Indian Hort. 45 (3 \& 4): 181-183.

Paul, M. C. and Mishra, R. R., 1994, Seed germination and seedling vigour of maize (Zea mays) as influenced by different fungicides. Crop Res. (Hissar), 7: 454-460.

Raikar, S. D., Vyakarnahal, B. S., Biradar, D. P., Deshpande, V. K., and Janagoudar, B. S., 2011, Effect of seed source, containers and seed treatment with chemical and biopesticide on storability of scented rice $\mathrm{Cv}$. Mugad sugandha Karnataka J. Agric. Sci., 24 (4) : 448-454.

Remya, J., 2007, Influence of vacuum packaging and long terms storage on quality in chilli. Powder, M. Sc.(Agri) Thesis, Univ. of Agric. Sci., Dharwad, Karnataka (India).

Samuel, T. A., Hussaini, A. M., Titilayo, A. and Ibrahim, K., 2008, Effect of Fusarian verticilloids, its metabolites and neem leaf extract on germination and vigour indices of maize (Zea mays L.). Afri. J. Btech., 7 (14): 2402-2406

Stibilj V., Kreft I., Smrkolj P., Osvald J. (2004) Enhanced selenium content in buckwheat (Fagopyrum esculentum Moench) and pumpkin (Cucurbita pepo L.) seeds by foliar fertilisation. European Food Research and Technology, 219:142-144.

\section{How to cite this article:}

Chelakani Srujana, Ajay Kumar Pandey and Chaurasia, A. K. 2020. Influence of Seed Treatments and Packaging Materials on Seed Quality Parameters during storage in Buck wheat (Fagopyrum esculentum L.). Int.J.Curr.Microbiol.App.Sci. 9(08): 1738-1744. doi: https://doi.org/10.20546/ijcmas.2020.908.200 